\title{
Age-related Changes in Astrocytes and Microvasculature in the Median Eminence of the Rat
}

\author{
Hiromi Ike ${ }^{1}$, Yoshitaka Tamada ${ }^{1}$, Mamoru Uemura ${ }^{1}$, Akihiko Ishihara ${ }^{2}$, \\ Fumihiko Suwa ${ }^{1}$ and Yasuhiko Ibata $^{3}$ \\ ${ }^{1}$ Department of Anatomy, Osaka Dental University, 8-1 Kuzuhahanazono-cho, Hirakata-shi, Osaka 573-1121, Japan, \\ ${ }^{2}$ Laboratory of Neurochemistry, Graduate School of Human and Environmental Studies, Kyoto University, Sakyo-ku, \\ Kyoto 606-8501, Japan and ${ }^{3}$ Kyoto Prefectural University of Medicine, Kawaramachi-Hirokoji, Kamigyo-ku, Kyoto \\ 602-0841, Japan
}

Received December 10, 2003; accepted February 16, 2004

Age-related changes in astrocytes, tanycytes and the microvasculature were investigated in the median eminence (ME) of the anterior hypothalamo-hypophyseal system of 2- and 24-month old rats by means of immunocytochemistry and scanning electron microscopy. A computer-assisted image processing system showed a significant increase of the glial fibrillary acidic protein (GFAP) immunoreactivity in the internal layer (IL) and the perivascular region of the external layer (EL) of the ME with age. However, no remarkable changes in either layer could be detected employing vimentin immunoreactivity. An immunoelectron microscopic study revealed that the number of the GFAP-immunoreactive processes markedly increased with age in regions surrounding the capillaries of the EL. Therefore, it may be considered that, in both layers, the astrocytic cell bodies and associated glial processes exhibit a significant increase with age. Investigation of the microvasculature under a scanning electron microscope demonstrated the development and the sinusoidal progress of the external capillary plexus with age, but these changes were not observed in the internal capillary network of the ME. In conclusion, the results of the present study showed a close correlation between the increase of the GFAP-immunoreactive astrocytes and the development of the external capillary plexus. In addition, there was a close correlation between the increase in the GFAP-immunoreactive astrocytes and the sinusoidal progress.

Key words: immunocytochemistry, median eminence, astrocyte, microvasculature, rat

\section{Introduction}

Many different types of cellular components, such as nerve endings and glial processes, have been identified in the median eminence (ME) of the mammalian species [21]. It is thought that these glial cells are involved in the regulation of the release of the releasing/inhibiting $(\mathrm{R} / \mathrm{I})$ hormones in the ME $[11,13,15-17,26]$.

Tanycytes and astrocytes have been identified in the ME [3, 4] by immunocytochemistry for vimentin (VIM) $[19,24]$ and glial fibrillary acidic protein (GFAP) $[5,22]$.

Correspondence to: Yoshitaka Tamada, D.D.S, Ph.D., Department of Anatomy, Osaka Dental University, 8-1 Kuzuhahanazono-cho, Hirakata-shi, Osaka 573-1121, Japan.

E-mail: tamada@cc.osaka-dent.ac.jp
Tanycytes are immunoreactive to both VIM and GFAP [24]. These cell bodies are located in the ependymal layer and send processes to the capillaries of the primary capillary plexus of the hypophyseal portal system [18] in the palisade layer and the perivascular region [7, 23]. Astrocytes are immunoreactive to GFAP and immunonegative to VIM [4]. Astrocytic cell bodies are mainly distributed in the subependymal layer and their processes have been observed throughout the ME [26].

In the ME, it has already been clarified morphologically that the GFAP-immunoreactive processes are in close contact with nerve endings such as the synapses, and a specific contact pattern is also exhibited between these processes and the basement membrane of the capillaries in the perivascular region [26]. The microvasculature of the $\mathrm{ME}$ in the anterior hypothalamo-hypophyseal system (HHS) 
was investigated in the rat by Murakami et al. [18]. However, very few studies have focused on the age-related morphological changes in glial cells and the microvasculature in the ME.

Therefore, we employed immunocytochemistry for VIM and GFAP, and the plastic injection method [20] in order to investigate the age-related changes that occurred in glial cells and the microvasculature in the ME.

\section{Materials and Methods}

\section{Experimental animals}

Twenty-four young adult male Sprague-Dawley (SD) rats ( 2 months old, young adult group, body weight; about $270 \mathrm{~g}$ ) and 20 aged male SD rats (24 months old, aged group, body weight; $870-980 \mathrm{~g}$ ) were used in the present study. All rats were purchased from Charles River Japan, Inc. (Atsugi City) and housed in a temperature-controlled room $\left(20^{\circ} \mathrm{C}\right)$. All rats were maintained in individual cages under a light/ dark cycle (light on at 0700 and light off at 1900) and were given free access to food and water.

The Committee of Animal Research, Kyoto Prefectural University of Medicine, approved the experiments done in the present study. The present study was conducted according to Guidelines for Animal Research at Osaka Dental University and the National Institute of Health (NIH) Guideline for the Care and Use of Laboratory Animals.

\section{Immunocytochemistry}

Light microscopic immunocytochemistry

Ten animals in each group were perfused under deep pentobarbital anesthesia $\left(40 \mathrm{mg} / \mathrm{kg}\right.$, i.p., $\left.\mathrm{Nembutal}^{\mathbb{R}}\right)$ via the left cardiac ventricle with $0.1 \mathrm{M}$ phosphate-buffered saline (PBS) and a fixative containing 4\% paraformaldehyde and $0.2 \%$ picric acid, which was adjusted to $\mathrm{pH} 7.4$ with $0.1 \mathrm{M}$ phosphate buffer (PB). Then, the brain of each animal was immediately removed. Tissue blocks, including the ME, were dissected from the hypothalamus and then post-fixed in the same fixative for $24 \mathrm{hr}$ at $4^{\circ} \mathrm{C}$. After fixation, the tissue blocks were immersed in $20 \%$ sucrose in $0.1 \mathrm{M} \mathrm{PB}$ for $24 \mathrm{hr}$ at $4^{\circ} \mathrm{C}$.

Serial frontal free-floating sections $(30 \mu \mathrm{m}$ in thickness) of the young adult and the aged groups were cut with a cryostat and stored in $0.1 \mathrm{M}$ PBS containing $0.1 \%$ Triton X100 for $24 \mathrm{hr}$ at $4^{\circ} \mathrm{C}$. Then, the sections were processed for the avidin-biotin complex (ABC) method using antibodies against VIM and GFAP. The free-floating sections were incubated in the anti-VIM and the anti-GFAP serums (VIM, dilution 1:1000, Sigma; GFAP, dilution 1:1000, DAKO) in $0.1 \mathrm{M}$ PBS containing $0.1 \%$ Triton-X 100 for $72 \mathrm{hr}$ at $4^{\circ} \mathrm{C}$. After the treatment, the sections were incubated in the secondary antibodies (VIM, biotinylated anti-mouse IgG; GFAP, biotinylated anti-rabbit IgG, Vector Lab. Inc.) overnight at $4^{\circ} \mathrm{C}$ and then were treated with the $\mathrm{ABC}$ solution (dilution 1:1000, Vector Lab. Inc.) overnight at $4^{\circ} \mathrm{C}$. They were exposed for $10 \mathrm{~min}$ at room temperature to a $50 \mathrm{mM}$ Tris-HCl buffer containing $0.01 \%$ 3-3'-diaminobenzidine
$4 \mathrm{HCl}(\mathrm{DAB})$ and $0.005 \% \mathrm{H}_{2} \mathrm{O}_{2}$. All specimens were marked to discriminate the individual animals, and all free-floating sections of the young adult and the aged groups were reacted in the same solution for the same amount of time. All sections were mounted on glass slides and examined under a light microscope.

The specificity of the anti-VIM and the anti-GFAP serums were confirmed by preabsorption with the respective antigens (1-100 mg), which completely abolished immunostaining.

\section{Quantification of immunocytochemistry and statistical analysis}

A computer-assisted image processing system was employed to investigate the quantitative analysis of VIM and GFAP immunoreactivity. The age-related changes in the expressions of VIM and GFAP in the ME were examined.

Ten serial frontal sections of the middle part of the ME were randomly selected for each animal and used to quantify the VIM and GFAP immunoreactivity in young adult and aged groups.

For expediency in the quantitative analysis, the ME was divided into sixteen blocks as shown in Figure 1. The optical density (OD) of the VIM and the GFAP immunoreactivity in each block was measured using NIH image software and the background OD was excluded from the ODs of each region. The OD values in 1, 4, 7, 10 and 13 blocks were summed up as those of the internal layer (IL), those in 2, 5, 8, 11 and 14 as the middle layer (ML) and those in 3, 6, 9, 12 and 15 as the external layer (EL). Furthermore, the values for individual layers were added up and defined as those of the total layer (TL).

The sections were digitized as gray-level images on a computer-assisted image processing system. Each pixel was quantified as 1 of 256 gray levels. A gray-level value of zero

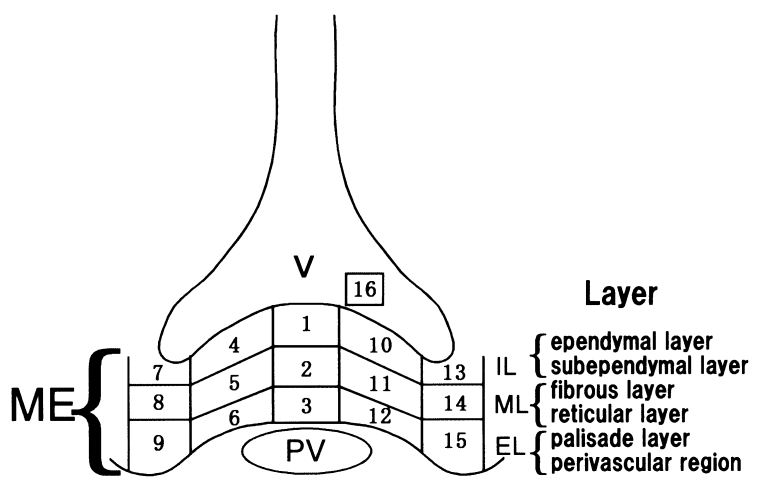

Fig. 1. Division of ME for quantitative analysis. The ME was expediently divided into sixteen blocks. The ODs in the IL, the ML, the $\mathrm{EL}$ and the TL were calculated according to the following formulae; $\mathrm{IL}=1+4+7+10+13$ (ependymal and subependymal layers); $\mathrm{ML}=2+5+$ $8+11+14$ (fibrous and reticular layers); $E L=3+6+9+12+15$ (palisade layer and perivascular region); $\mathrm{TL}=\mathrm{IL}+\mathrm{ML}+\mathrm{EL}$; Background, 16; IL, internal layer; ML, middle layer; EL, external layer; TL, total layer; V, third ventricle; ME, median eminence. 
was equivalent to $100 \%$ transmission of light and that of 255 was equivalent to $0 \%$ transmission. The mean $\mathrm{OD}$ values of all pixels were determined using a calibration tablet which has 21 steps of gradient density ranges and diffused density values.

The values for individual layers were represented as mean \pm standard deviation. A one-way analysis of variance (ANOVA) was used to test for statistical significance. Fisher's post hoc tests were used to determine significant group differences $(\mathrm{n}=10, \mathrm{p}<0.001, \mathrm{p}<0.05)$.

\section{Electron microscopic immunocytochemistry}

To examine the fine structure of the ME, five animals in each group were euthanized by deep pentobarbital anesthesia $\left(40 \mathrm{mg} / \mathrm{kg}\right.$, i.p., Nembutal $\left.{ }^{\mathbb{R}}\right)$, and decapitated to prevent the destruction of the capillary in the ME caused by the pressure of perfusion with the fixative. The brain of each animal was removed as quickly as possible, and the tissue blocks of the hypothalamus, including the ME, were immersed in a fixative containing $4 \%$ paraformaldehyde, $0.2 \%$ picric acid and $0.35 \%$ glutaraldehyde for $72 \mathrm{hr}$ at $4^{\circ} \mathrm{C}$. Then, they were fixed in a fixative without glutaraldehyde for $24 \mathrm{hr}$ at $4^{\circ} \mathrm{C}$, and placed in 0.1 M PBS. Serial frontal sections (40 $\mu \mathrm{m}$ in thickness) of the tissue were cut with a microslicer in 0.1 M PBS from the tissue blocks, including the ME. For the electron microscopic immunocytochemistry, the sections were treated similarly to those for the light microscopic immunocytochemistry. After confirmation of the VIM- and the GFAP-immunoreactive elements under a light microscope, the sections were additionally fixed in $1 \% \mathrm{OsO}_{4}$ solution for $1 \mathrm{hr}$ at $4^{\circ} \mathrm{C}$, dehydrated through a graded series of acetone and embedded in an Epon 812 mixture. Semithin sections were examined under a light microscope. Ultrathin sections were then stained with uranyl acetate and examined under a transmission electron microscope (JEM200CX, JEOL).

\section{Microvasculature corrosion casts by the plastic injection method}

Nine animals from the young adult group and five from the aged group were used for the investigation of the microvasculature in the ME. Under deep anesthesia with diethyl ether and pentobarbital $\left(40 \mathrm{mg} / \mathrm{kg}\right.$, i.p., Nembutal $\left.{ }^{\circledR}\right)$, they were perfused with $0.5 \mathrm{ml}$ heparinized saline solution $\left(5,000 \mathrm{IU} / \mathrm{I}\right.$, Novo Heparin $\left.{ }^{\circledR}\right)$ via the femoral vein. Ten minutes later, they were euthanized by bleeding from the femoral artery. After the confirmation of death, the chests of the animals were opened, and the thoracic aorta and the inferior vena cava were ligated. Next, a metallic cannula (1.5 $\mathrm{mm}$ in diameter) was inserted in the ascending aorta via the left ventricle, and the aorta was ligated and fixed. According to the plastic injection method [20], at first $15 \mathrm{ml}$ of acrylic plastic (methyl methacrylate) with a low viscosity was injected, and then $5 \mathrm{ml}$ with a high viscosity was injected. After the injection, the cannula was immediately extracted and the ascending aorta was ligated. To promote the polymerization of the plastic, the injected specimen was kept at $50^{\circ} \mathrm{C}$ in a water bath. After polymerization, the soft tissue of the head was removed with a surgical knife to improve the effect of digestion in the preparation of the specimen, and the skull was decalcified with $10 \%$ formalin solution including $5 \%$ hydrochloric acid.

Five decalcified heads from each group were cut sagittally to the vicinity of the median plane of the ME with a microtome under freezing conditions. The specimens were digested in the $5 \%$ sodium hypochlorite solution and a $10 \%$ sodium hydroxide solution, and then prepared for the microvascular corrosion cast of the pituitary system observed from the median plane.

In order to examine the arteries of the blood supply and the microvasculature of the whole ME in three dimension, the soft tissue of four of the nine young adult rats was digested in the same solution after the decalcification as mentioned above. Two of these animals were prepared as specimens for the observation of the artery of the blood supply to the ME, and the other two animals as specimens for the observation of the capillary plexus from the dorsal side.

Each specimen was placed on a sample carrier with conduction tape and conduction paste (DOTITE ${ }^{\circledR}$ ), coated with gold with an ion-coater (JFC-1500, JEOL) and examined under a scanning electron microscope (SEM) (JSM5500 , JEOL; working distance, $48 \mathrm{~mm}$; acceleration voltage, $5-15 \mathrm{kV})$.

\section{Results}

\section{Light microscopic immunocytochemistry}

VIM-immunoreactive tanycytic cell bodies were located in the ependymal layer of the IL just below the third ventricle. Tanycytic processes were observed to extend radially toward the floor of the brain and around the capillaries in the EL. In the mediobasal hypothalamus, except for the ME regions, tanycytic processes were found running more horizontally (Fig. 2a). In a comparison of the young adult and the aged groups, no prominent morphological differences were observed. The distributions of these tanycytic processes were similar for the two groups in spite of the age-related changes in the size of the ME (Fig. 2b).

However, the distribution of the GFAP in the ME was greatly different from that of the VIM. The GFAP-immunoreactive tanycytic cell bodies were located in the ependymal layer of the IL, and the GFAP-immunoreactive processes ran toward the EL. The GFAP-immunoreactive astrocytic cell bodies were also found in the subependymal layer just below the ependymal layer, and the GFAP-immunoreactive processes ran toward the EL (Fig. 2c). The expression of the GFAP in the ME of the aged group showed a remarkable increase in comparison with the young adult group. Especially, many astrocytes and their processes were observed to extend from the subependymal layer in the IL to the perivascular regions in the EL (Fig. 2d). 

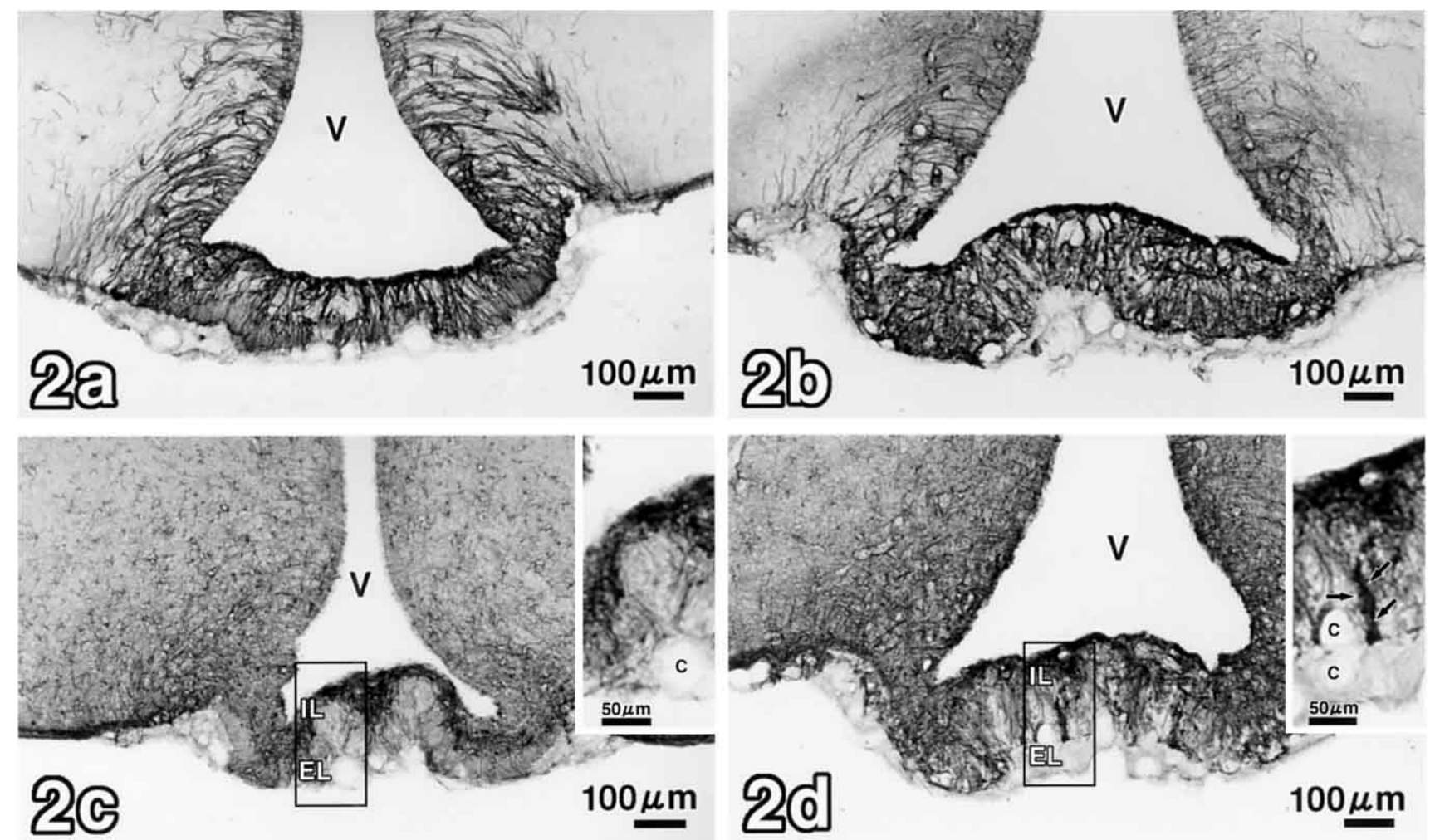

Fig. 2. Light microscopic immunocytochemistry for VIM (a, young adult group; b, aged group) and GFAP (c, young adult group; d, aged group). a, b: No prominent changes were observed in the distribution of VIM-immunoreactive elements between the young adult and aged groups. c, d: GFAP-immunoreactive elements in the aged group showed a remarkable increase in comparison with the young adult group. The square areas were enlarged in the insets. In the aged group, many GFAP-immunoreactive elements (arrows) were observed from the IL to the EL. C, capillary.

\section{Quantitative analysis of the VIM and the GFAP immunoreac- tivity in the $M E$}

In a comparison of the young adult and the aged groups, no significant differences in the VIM-immunoreactive density in the tanycytes were found in any of the layers (young adult group IL, 213.16 \pm 7.60 ; ML, 182.14 \pm 2.47 ; EL, 192.49 \pm 3.47 , aged group IL, 214.29 $\pm 13.60 ;$ ML,

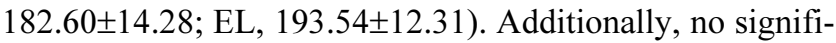
cant differences were detected in the ODs of the TL in the ME (TL young adult group, 195.93 \pm 4.41 ; aged group, 196.81土13.08) (Fig. 3a).

In contrast, the GFAP immunoreactivity showed significant differences in both groups (Fig. 3b). In both the IL and the EL, the GFAP immunoreactivity was significantly higher in the aged group than in the young adult group (young adult group IL, 190.24 \pm 4.35 ; EL, 160.07 \pm 5.88 ; aged group

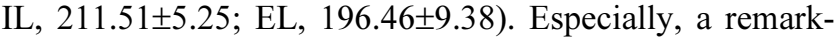
able increase in GFAP immunoreactivity was observed in the EL. However, no significant change was observed in the ML (ML young adult group, 187.74 \pm 12.96 ; aged group, 200.01 \pm 6.58 ). Adding up the ODs for all layers, the GFAP immunoreactivity showed significant age-related changes

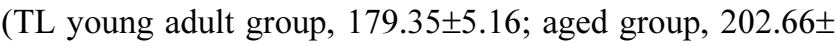
6.62) (Fig. 3b).

\section{Immunoelectron microscopy}

Immunoelectron microscopic findings for the young adult group were previously reported by Tamada et al. [26].

In the aged group, astrocytic cell bodies were observed abundantly, not only in the subependymal layer, but also in the reticular layer of the ML. Especially, cell bodies showing a strong GFAP immunoreactivity were identified in the vicinity of the capillary invading the reticular layer (Fig. 4a). Moreover, the GFAP-immunoreactive processes were distributed around the abundant capillary of the hypophyseal portal system in the perivascular region. In the aged group, these processes showed a tendency to increase, in comparison with the young adult group. In addition, the size of the area in which these processes were detected around the capillary remarkably increased with age (Fig. 4b).

\section{Microvasculature investigation employing SEM to determine blood supply to the ME in the young adult group}

The microvasculature of the ME was observed on the ventral side of the third ventricle (Figs. 5, 6a). The microvasculature of the adenohypophysis was observed behind the microvasculature of the ME (Fig. 5). The hypophyseal portal vessel, consisting of about ten venules, was observed connecting with both of the microvasculatures (Fig. 5). Functioning as blood supply to the ME, the anterior and 

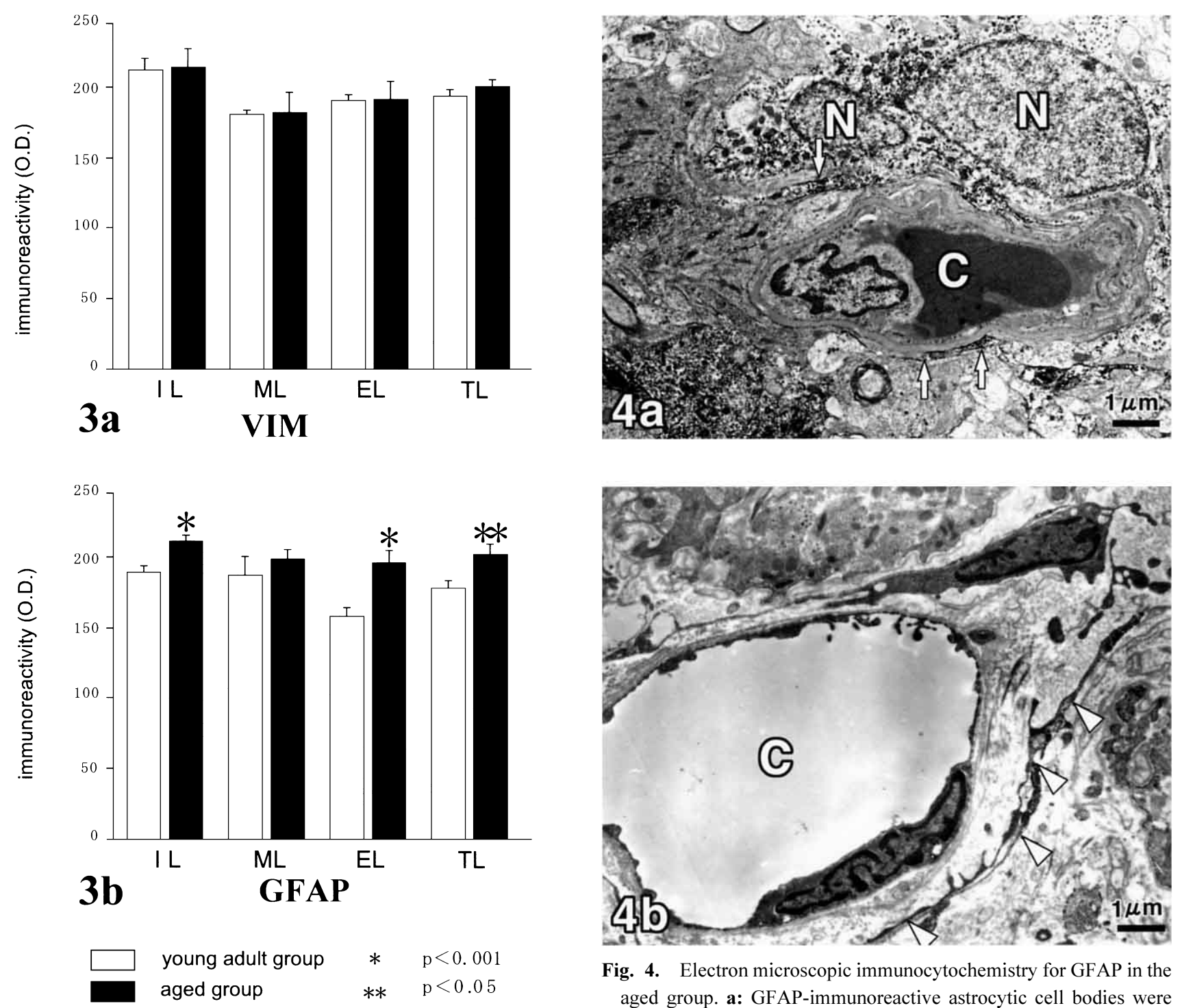

Fig. 4. Electron microscopic immunocytochemistry for GFAP in the aged group. a: GFAP-immunoreactive astrocytic cell bodies were observed in the vicinity of the capillary in the reticular layer of ML. Many GFAP-immunoreactive processes (arrows) were identified around the capillary. b: There was a wide spread of astrocytic processes (arrowheads) ending around the capillary in the perivascular region of EL. N, nuclei of astrocytic cell bodies. observed in GFAP immunoreactivity in IL, EL and TL of the aged group, in comparison with that of the young adult group $(n=10$, $\left.{ }^{*} \mathrm{p}<0.001,{ }^{*} \mathrm{p}<0.05\right)$. Error bars indicate the standard deviation of the mean.

the middle hypophyseal arteries arising from the internal carotid artery were also observed (Fig. 5). From the lateroventral view, the accessory middle hypophyseal artery could not be observed, because this artery was located dorsal to the microvasculature of the adenohypophysis (Fig. 5).

\section{Microvasculature in the ME of the young adult group and the aged group}

Young adult group

The microvasculature of the ME consisted of the external capillary plexus and the internal capillary network (Fig. 6a).

External capillary plexus

The external capillary plexus was located in the EL and the ML (Fig. 7a). The middle part of the ME was the thickest in the dorso-ventral height (about $150 \mu \mathrm{m}$ ) (Fig. 7a). This plexus was composed of a capillary bed and capillary loops, and both were distinguished clearly (Figs. 6b, 7a).

The capillary bed, composed of many sinusoidal capillaries (6-20 $\mu \mathrm{m}$ in diameter), was congregated (Figs. 6b, 7a). It was located in the EL (Fig. 7a) and in close contact with the dorsal side of the hypophyseal portal vessels, which ran beneath the ventral surface of the ME (Fig. 7a).

Capillary loops projected to the dorsal side of the capillary bed (Figs. 6b, 7a). In addition, capillary loops were distinguished into simple low capillary loops (LCLs) in the EL 


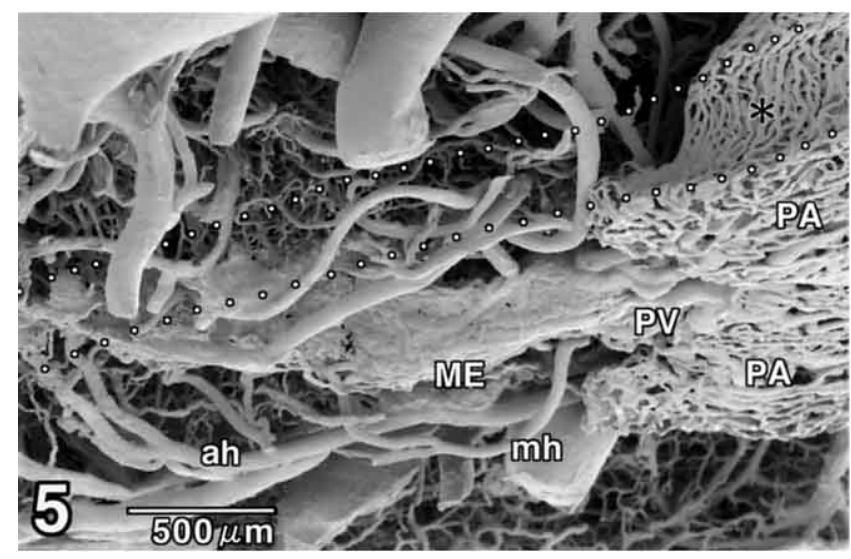

Fig. 5. Microvasculature of the hypothalamo-hypophyseal system in the young adult group (infero-lateral view). ME was located in the anterior region of the adenohypophysis (PA). ah, anterior hypophyseal artery; mh, middle hypophyseal artery; PV, hypophyseal portal vessels; *, impress of removed internal carotid artery; two dotted lines, removed internal carotid artery.

and the ML (Figs. 6b, 7a) and complicated high capillary loops (HCLs) in the ML (Figs. 6b, 7a). The LCLs (46 $\mu \mathrm{m}$ in height) were simple hairpin loops, which were composed of one or two short ascending crura (6-11 $\mu \mathrm{m}$ in diameter) and one short descending crus $(6-16 \mu \mathrm{m}$ in diameter) (Figs. 6b, $7 \mathrm{a})$. The HCLs (85-100 $\mu \mathrm{m}$ in height) showed a characteristic columnar or pyramidal shape when viewed as a whole (Figs. 6b, 7a). The tips of the HCLs bulged in a spherical or hemispherical form (Figs. 6b, 7a). The HCLs consisted of 2 to 4 ascending crura (5-14 $\mu \mathrm{m}$ in diameter) and descending crura (7-14 $\mu \mathrm{m}$ in diameter) (Figs. 6b, 7a).

\section{Internal capillary network}

The internal capillary network was located in the IL of the ME (Fig. 7a), and a small number of sinusoidal capillaries ran on horizontally, meandering on the dorsal side of the external capillary plexus (Fig. 6a, b). The network consisted of only two or three meshes of anastomotic capillaries (Fig. $6 \mathrm{a}, \mathrm{b})$. These sinusoidal capillaries were both thick (20-30 $\mu \mathrm{m}$ in diameter) and thin (10-12 $\mu \mathrm{m}$ in diameter), and were more the thick capillaries than the thin (Fig. 6a, b). The capillaries of the internal capillary network communicated with those of the capillary bed or the HCLs of the external capillary plexus (Figs. 6b, 7a).

\section{Hypophyseal portal vessels}

The hypophyseal portal vessels were venules (about 43 $\mu \mathrm{m}$ in diameter) running rostro-caudally, and were located on the ventral side of the ME (Fig. 7a, c). These portal vessels communicated with the capillaries of the capillary bed of the external capillary plexus (Fig. 7a).

\section{Aged group}

The external capillary plexus and the internal capillary network were observed in the aged group (Fig. 7b) as well as
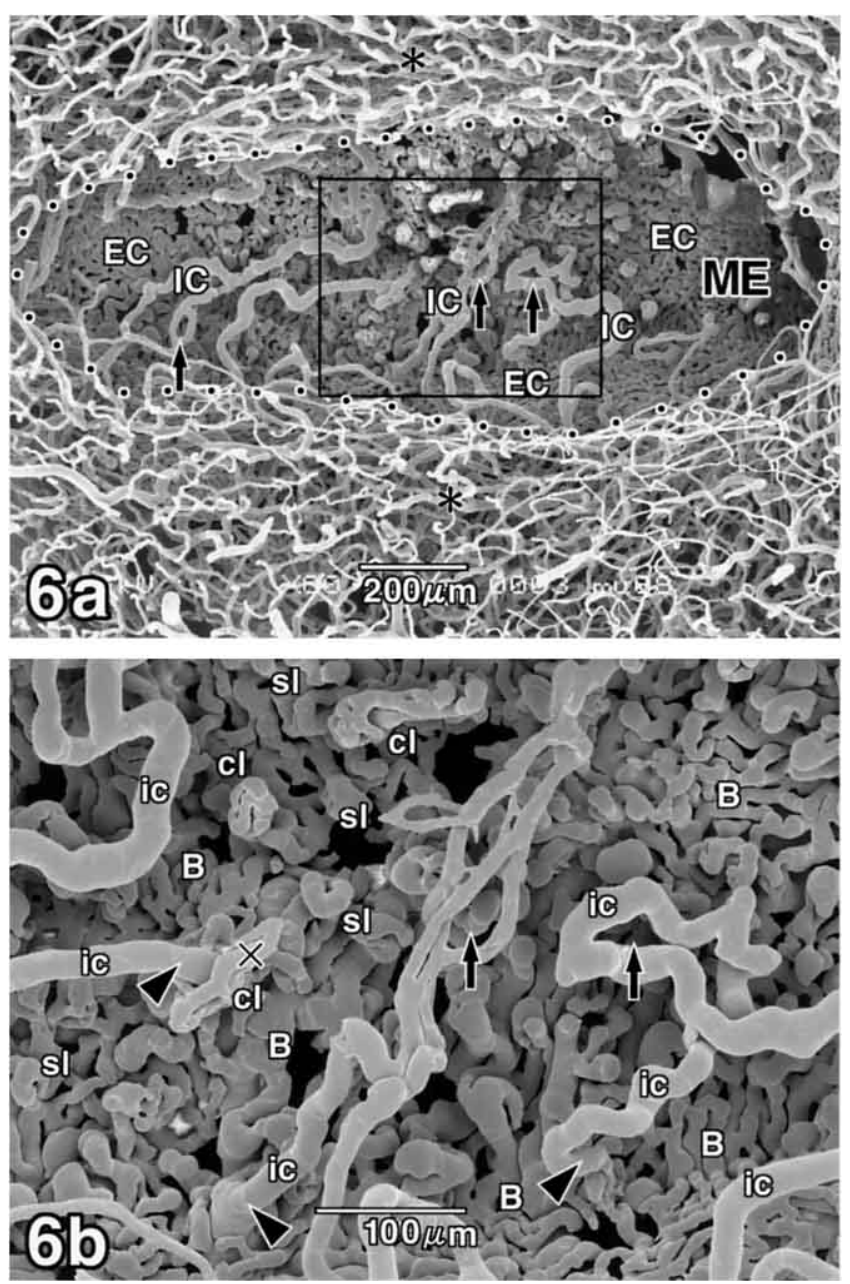

Fig. 6. The microvasculature of the ME in the young adult group (dorsal view from the third ventricle). a: The microvasculature of the ME consisted of the internal capillary network (IC) on the dorsal side and the external capillary plexus (EC) on the ventral side. b: Close-up of the square area in a. The external capillary plexus consisted of the capillary bed (B) and, simple low capillary loops (sl) and complicated high capillary loops $(\mathrm{cl})$. These loops projected into the dorsal side from the capillary bed. The capillary bed consisted of many sinusoidal capillaries. Within the dotted line, third ventricle; *, microvasculature of the hypothalamus; arrows, a few meshes of capillaries of the internal capillary network; ic, sinusoidal capillaries of the internal capillary network; $\times$, communication between the internal capillary network and the complicated high capillary loops; arrowhead, communication between the internal capillary network and the capillary bed.

in the young adult group (Figs. 6a, 6b, 7a). Prominent morphological changes were observed in the external capillary plexus (Fig. 7b).

\section{External capillary plexus}

The external capillary plexus was located in the EL and the ML of the ME (Fig. 7b). The middle part of the ME was the thickest in dorso-ventral thickness (about $230 \mu \mathrm{m}$ ), and was enlarged about 1.5 times (Fig. 7b) in comparison with 

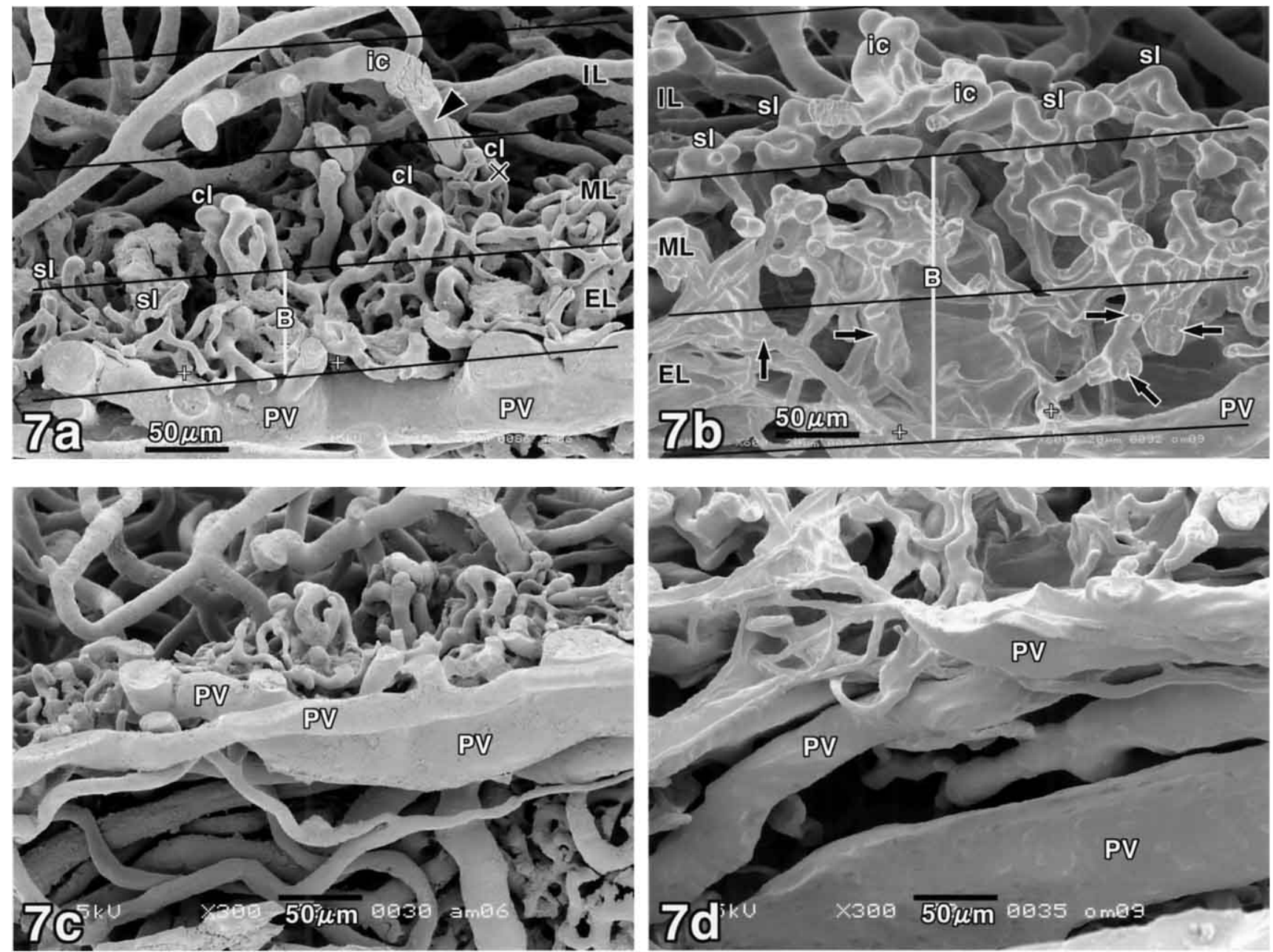

Fig. 7. The internal capillary network, the external capillary plexus and the hypophyseal portal vessels in the microvasculature of the ME (a and c, young adult group; b and d, aged group). a: The capillary bed (B) was located in the EL (the white line shows the height of B). Simple low capillary loops (sl) were located in the EL and the ML, and had a simple hairpin appearance. Complicated high capillary loops (cl) were located in the ML, and form columnar or pyramidal shapes. The internal capillary network was located in the IL and its capillaries (ic) appeared sinusoidal (midline dissected view). b: The capillary bed (B) developed from the EL to the ML (the white line shows the height of B) (compare with a). Only the simple low capillary loops (sl) were seen in the IL. The sinusoidal progress (arrows) of the capillaries in the EL had advanced. The internal capillary network was located in the IL and its capillaries (ic) had not changed in thickness. (midline dissected view). c: The hypophyseal portal vessels (PV) ran rostro-caudally on the ventral surface of the external capillary plexus. The diameter of these portal vessels was about $43 \mu \mathrm{m}$. (ventral view of a). d: The diameter of the hypophyseal portal vessels (PV) had grown about two-fold, $88 \mu \mathrm{m}$ more than that seen in the young adult group. (ventral view of b). IL, internal layer; ML, middle layer; EL, external layer; +, communication between the capillary bed and the hypophyseal portal vessels; arrowhead, communication between the internal capillary network and the capillary bed; $\times$, communication between the internal capillary network and the complicated high capillary loops; arrows, the sinusoidal progress; the black lines indicate the border of each layer.

that (about $150 \mu \mathrm{m}$ ) of the young adult group (Fig. 7a). The external capillary plexus between the capillary bed and the capillary loops was distinguished clearly (Fig. 7b).

It was observed that the capillary bed developed dorsally in comparison with that of the young adult group (Fig. 7a), and was widely distributed from the EL to the ML in the ME. This bed was composed of sinusoidal capillaries (6-30 $\mu \mathrm{m}$ in diameter) (Fig. 7b).

Capillary loops were located in the IL on the dorsal side of the developed capillary bed (Fig. 7b). These loops were only low loops (22-32 $\mu \mathrm{m}$ in height) composed of two ascending crura (7-14 $\mu \mathrm{m}$ in diameter) and a descending crus (about $9 \mu \mathrm{m}$ in diameter), and were not columnar or pyramidal HCLs (Fig. 7b), as was observed in the young adult group (Fig. 7a).

\section{Internal capillary network}

The internal capillary network was located in the IL and consisted of two or three meshes of anastomotic capillaries (Fig. 7b). This was observed as well as in the young 
adult group (Fig. 7a).

The sinusoidal capillaries composing this network were 19-30 $\mu \mathrm{m}$ and 10-14 $\mu \mathrm{m}$ in diameter, which was not widely different (Fig. 7b) from those of the young adult group (Fig. 7a). However, these capillaries ran closely along the dorsal surface of the external capillary plexus (Fig. 7b).

\section{Hypophyseal portal vessels}

The hypophyseal portal vessels were about $88 \mu \mathrm{m}$ in diameter, and were enlarged about twice in size (Fig. 7d), compared with that of the young adult group (Fig. 7c).

\section{Discussion}

\section{Experimental procedures in the present study}

The ME was expediently divided into sixteen blocks for quantitative analysis of the GFAP and the VIM immunoreactivity. This division method was employed in order to examine the relationship between the age-related changes in immunoreactivity and the microvasculature in the IL, ML and EL. In this division method, the ependymal and the subependymal layers corresponded to the IL, the fibrous and the reticular layers to the ML, and the palisade layer and the perivascular region to the EL. After the measurement of OD in each block, the density was examined in contrast with the microvasculature in each layer.

To minimize the technical variations of the age-related changes of the GFAP- and the VIM-immunoreactivity in the quantitative analysis using a computer-assisted image processing system, all the free-floating sections of the young adult and the aged groups were marked and processed simultaneously in the same reactive solution for the same amount of time.

\section{Age-related changes in glial cells in the $M E$}

It was clarified that there was an age-related increase in the number of astrocytes [14]. There were also age-related hypertrophic changes in the astrocytes $[1,2,25]$. Zoli et al. suggested that these changes were one of the age-related phenomena in the hypothalamus [28]. However, very few studies have focused on the aged-related changes in glial cells in the ME. The quantitative analysis showed a significant age-related increase in GFAP immunoreactivity in the IL and the EL, but no age-related changes in the VIM immunoreactivity. Our quantitative data on the GFAP immunoreactivity suggested an age-related increase in astrocytes in the IL and the EL of the ME.

In contrast, experimental brain injuries produced the following results: (1) an increase of GFAP and an induction of its mRNA [8, 27], however, no proliferation of astrocytes was observed [10], (2) astrocytes produced several kinds of neurotrophic factors, such as nerve growth factor (NGF) [6, $9,12]$. These studies suggested that the number of astrocytes did not increase, but that the expression of GFAP and NGF increased in the astrocytic cell bodies with aging and injury. Additionally, these studies suggested that GFAP and NGF played important roles in supporting neuronal survival.
Moreover, in an in vitro study using the co-culture systems of glial cells and neurons in the ME, it was revealed that aged glial cells had the capacity to support neurite outgrowth and the survival of neurons [4]. Based on this experimental evidence, the age-related increase in GFAP in astrocytes of the ME was considered to contribute greatly to neuronal survival.

\section{Age-related changes in microvasculature in the ME}

Murakami et al. investigated the microvasculature of the rat HHS according to animal body weight [18]. Their research work, however, makes no mention with regard to the age of animals. In the present study, we clarified both the age (24 month-old) and body weights (870-980 g) of the experimental animals, and investigated the morphological changes in the microvasculature in the ME of aged rats with heavy weight body as compared with those used by Murakami et al.

Murakami et al. divided the microvasculature of the $\mathrm{ME}$ and the infundibular stalk in the HHS into the subependymal capillary network and the capillary bed. In the present study, we investigated only the microvasculature of the $\mathrm{ME}$, and did not investigate the infundibular stalk. We regarded the subependymal capillary network as the internal capillary network, and the capillary bed as the external capillary plexus. In addition, Murakami et al. included the capillary loops in the capillary bed, while we divided the external capillary plexus into the capillary bed and the capillary loops. The reason why we employed this division was on the basis of the following evidence: Murakami et al. reported age-related changes in the capillary loops of the capillary bed, while we identified age-related changes in both the capillary bed and the capillary loops of the external capillary plexus. Therefore, we discussed age-related changes in the internal capillary network and the external capillary plexus in order.

The internal capillary network was located in the IL of both groups and it was an incomplete capillary network composed of only two or three meshes of anastomotic capillaries. In regard to the subependymal capillary network, Murakami et al. mentioned only that it was composed of anastomotic capillaries, and there was no mention of agerelated changes in this network. In the present study, no age-related changes in the capillaries of this network were observed.

However, we found that the external capillary plexus developed with age according to the following positional changes. With advanced age, the capillary bed developed from the EL in the young adult group to the EL and the ML in the aged group, and as this development took place, a change was found from the LCLs and the columnar HCLs in the young adult group to the low loops in the aged group. Subsequently, both capillary loops became the low loops.

Moreover, with advanced age, the capillaries became thicker. In the aged group, the diameter of the capillaries in the EL were from $20 \mu \mathrm{m}$ to $30 \mu \mathrm{m}$ thicker than that in the young adult group, therefore, it was thought that sinusoidal 
changes in the capillaries had advanced. Additionally, in the aged group, the external capillary plexus grew to a height about 1.5 times that seen in the young adult group. The diameter of the hypophyseal portal vessel became thicker in order to receive the increased blood flow from the external capillary plexus. From these facts, it was conjectured that the age-related changes in the external capillary plexus influences the age-related changes in the microvasculature of the hypophyseal portal vessels.

\section{Relation between astrocyte and microvasculature in advanced age}

In the IL, we clarified an age-related increase in the OD in GFAP immunoreactivity. It was suggested that the number and the enlargement of astrocytic cell bodies occurred in the subependymal layer with age [2]. As no changes were observed in the internal capillary network of the IL in either group, it was therefore thought that there was no correlation between the increase in the number of astrocytic cell bodies and the capillary network. However, it was conjectured that the number of astrocytes increased in the IL in order to receive the blood supply from the low loops of the external capillary plexus in the IL in the aged group.

In the ML, no significant increase in GFAP immunoreactivity was detected between the two groups. We clarified the age-related appearance of astrocytic cell bodies in the reticular layer of the $\mathrm{ML}$ in an electron microscope study. However, the number of astrocytic cell bodies that appeared in the ML of the aged group was very few, and so we concluded that they did not reflect the increase in OD in GFAP immunoreactivity. Additionally, it was also conjectured that the external capillary plexus developed into the ML in order to enhance the blood supply with these astrocytic cell bodies.

In the EL, it was shown that the age-related increase in GFAP immunoreactivity was due to the increased number of the GFAP-immunoreactive processes surrounding the capillaries. Furthermore, it was considered that the sinusoid of the capillaries progressed according to the increased number of these astrocytic processes with age.

In conclusion, the results of this study showed a close correlation between the increase of the GFAP-immunoreactive astrocytes and the development of the external capillary plexus. There was also a close correlation between the increase in the GFAP-immunoreactive astrocytes and the sinusoidal progress.

It is of importance to further elucidate what morphological and structural changes occur in the microvasculature in the ME after the dynamic changes of the astrocyte, and nerve processes and endings which may be induced by neuroactive substances such as R/I hormones, catecholamines and so on.

\section{Acknowledgments}

The authors wish to thank Dr. Michinori Hasegawa (Fuso Pharmaceutical Company, Osaka, Japan) for provid- ing aged rats.

\section{References}

1. Bjorklund, H., Eriksdotter-Nilsson, M., Dahl, D., Rose, G., Hoffer, B. and Olson, L. (1985) Image analysis of GFA-positive astrocytes from adolescence to senescence. Exp. Brain Res. 58; $163-170$.

2. Brawer, J. R. and Walsh, R. J. (1982) Response of tanycytes to aging in the median eminence of the rat. Am. J. Anat. 163;247256.

3. Chauvet, N., Parmentier, M. L. and Alonso, G. (1995) Transected axon of adult hypothalamus-neurohypophysial neurons regenerate along tanycytic processes. J. Neurosci. Res. 41; 129-144.

4. Chauvet, N., Privat, A. and Alonso, G. (1996) Aged median eminence gilal cells cultures promote survival and neurite outgrowth of cocultured neurons. Glia 18; 211-223.

5. Eng, L. F., Vanderhaeghen, J. J., Bignami, A. and Gerstl, B. (1971) An acidic protein isolated from fibrous astrocytes. Brain Res. 28; 351-354.

6. Ferrara, N., Ousley, F. and Gospodarowicz, D. (1988) Bovine brain astrocytes express basic fibroblast growth factor, a neurotrophic and angiogenetic mitogen. Brain Res. 462; 223-232.

7. Flament-Durand, J. and Brion, J. P. (1985) Tanycytes: morphology and functions: A review. Int. Rev. Cytol. 96; 121-155.

8. Fonseca, C. G., Green, C. R. and Nicholson, L. F. (2002) Upregulation in astrocytic connexin 43 gap junction levels may exacerbate generalized seizures in mesial temporal lobe epilepsy. Brain Res. 929; 105-116.

9. Furukawa, S., Furukawa, Y., Satoyoshi, E. and Hayashi, K. (1986) Synthesis and secretion of nerve growth factor by mouse astroglial cells in culture. Biochem. Biophys. Res. Commun. 136; 57-63.

10. Graeber, M. B., Lopez-Redondo, F., Ikoma, E., Ishikawa, M., Imai, Y., Nakajima, K., Kreutzberg, G. W. and Kohsaka, S. (1998) The microglia/macrophage response in the neonatal rat facial nucleus following axotomy. Brain Res. 813; 241-253.

11. Hatton, G. I., Perlmutter, L. S., Salm, A. K. and Tweedle, C. D. (1984) Dynamic neuronal-glial interactions in hypothalamus and pituitary: implications for control of hormone synthesis and release. Peptides 5 (Suppl 1); 121-138.

12. Ishikawa, R., Nishikori, K. and Furukawa, S. (1991) Appearance of nerve growth factor and acidic fibroblast growth factor with different time courses in the cavity-lesioned cortex of the rat brain. Neurosci. Lett. 127; 70-72.

13. King, J. C. and Letourneau, R. J. (1994) Luteinizing hormonereleasing hormone terminals in the median eminence of rats undergo dramatic changes after gonadectomy, as revealed by electron microscopic image analysis. Endocrinology 134; 13401351.

14. Kohama, S. G., Goss, J. R., Finch, C. E. and McNeill, T. H. (1995) Increases of glial fibrillary acidic protein in the aging female mouse brain. Neurobiol. Aging 16; 59-67.

15. Kozlowski, G. P. and Coates, P. W. (1985) Ependymoneuronal specializations between LHRH fibers and cells of the cerebroventricular system. Cell Tissue Res. 242; 301-311.

16. Langub, M. C., Jr. and Watson, R. E., Jr. (1992) Estrogen receptor-immunoreactive glia, endothelia, and ependyma in guinea pig preoptic area and median eminence: electron microscopy. Endocrinology 130; 364-372.

17. Montagnese, C., Poulain, D. A., Vincent, J. D. and Theodosis, D. T. (1988) Synaptic and neuronal-glial plasticity in the adult oxytocinergic system in response to physiological stimuli. Brain Res. Bull. 20; 681-692.

18. Murakami, T., Kikuta, A., Taguchi, T., Ohtsuka, A. and Ohtani, O. (1987) Blood vascular architecture of the rat cerebral hypo- 
physis and hypothalamus. A dissection/scanning electron microscopy of vascular casts. Arch. Histol. Jpn. 50; 133-176.

19. Naito, T., Yoshioka, H. and Yasuda, Y. (2002) Migration and proliferation of motile immature glial cells in the developing cerebral cortex of infantile rat. Acta Histochem. Cytochem. 35; 407-416.

20. Ohta, Y., Okuda, H., Suwa, F., Okada, S. and Toda, I. (1990) Plastic injection method for preparing microvascular corrosion casts for SEM and its practical application. Okajimas Folia Anat. Jpn. 66; 301-311.

21. Rodriguez, E. M., Gonzalez, C. B. and Delannoy, L. (1979) Cellular organization of the lateral and postinfundibular regions of the median eminence in the rat. Cell Tissue Res. 201; 377-408.

22. Sakai, H., Takeuchi, Y., Kawano, H., Matsushita, H., Yamazoe, I and Sugimoto, T. (2001) FK506 itself does not demonstrate neurotoxicity in the mouse brain. Acta Histochem. Cytochem. 34; 349-355.

23. Scott, D. E. and Paull, W. K. (1979) The tanycyte of the rat median eminence. I. Synaptoid contacts. Cell Tissue Res. 200; 329-334.

24. Shaw, G., Osborn, M. and Weber, K. (1981) An immunofluorescence microscopical study of the neurofilament triplet proteins, vimentin and glial fibrillary acidic protein within the adult rat brain. Eur. J. Cell Biol. 26; 68-82.

25. Sloane, J. A., Hollander, W., Rosene, D. L., Moss, M. B., Kemper, T. and Abraham, C. R. (2000) Astrocytic hypertrophy and altered GFAP degradation with age in subcortical white matter of the rhesus monkey. Brain Res. 862; 1-10.

26. Tamada, Y., Hayashi, S., Munekawa, K., Tanaka, M., Ikeda, T., Inoue, K. and Ibata, Y. (1997) Morphological interactions between glial fibrillary acidic protein (GFAP)-like immunoreactive elements and lutenizing hormone releasing hormone (LHRH)like immunoreactive nerve endings in the median eminence of the rat-Double labeling immunoelectron microscopic study-. Acta Histochem. Cytochem. 30; 517-523.

27. Tanaka, H., Araki, M. and Masuzawa, T. (1991) Differential response of three astrocyte-specific proteins to fimbrial transection of the rat brain: Immunohistochemical observations with antibodies against glial fibrillary acidic protein, glutamine synthetase and S-100 protein. Acta Histochem. Cytochem. 24; $11-19$.

28. Zoli, M., Ferraguti, F., Frasoldati, A., Biagini, G. and Agnati, L. F. (1995) Age-related alterations in tanycytes of the mediobasal hypothalamus of the male rat. Neurobiol. Aging 16; 77-83. 